\title{
Liposome accumulation in irradiated tumors display important tumor and dose dependent differences
}

Hansen, Anders Elias; Fliedner, Frederikke Petrine; Henriksen, Jonas Rosager; Jørgensen, Jesper Tranekjær; Clemmensen, Andreas Ettrup; Børresen, Betina; Elema, Dennis Ringkjøbing; Kjær, Andreas ; Andresen, Thomas Lars

Published in:

Nanomedicine: Nanotechnology, Biology and Medicine

Link to article, DOI:

10.1016/j.nano.2017.08.013

Publication date:

2018

Document Version

Peer reviewed version

Link back to DTU Orbit

Citation (APA):

Hansen, A. E., Fliedner, F. P., Henriksen, J. R., Jørgensen, J. T., Clemmensen, A. E., Børresen, B., Elema, D. R., Kjær, A., \& Andresen, T. L. (2018). Liposome accumulation in irradiated tumors display important tumor and dose dependent differences. Nanomedicine: Nanotechnology, Biology and Medicine, 14, 27-34. https://doi.org/10.1016/j.nano.2017.08.013

\section{General rights}

Copyright and moral rights for the publications made accessible in the public portal are retained by the authors and/or other copyright owners and it is a condition of accessing publications that users recognise and abide by the legal requirements associated with these rights.

- Users may download and print one copy of any publication from the public portal for the purpose of private study or research.

- You may not further distribute the material or use it for any profit-making activity or commercial gain

- You may freely distribute the URL identifying the publication in the public portal 
External beam radiation therapy (RT) is a central part of the treatment regimen for more than half of all cancer patients. Liposomal drug delivery systems that carry radio-senzitizers to

Conflict of interest: There are no conflicts of interest.

Funding: The support from the European Research Council, the Novo Nordisk Foundation, the Lundbeck Foundation, the Innovation Fund Denmark, the Research Council of Independent Research, the Svend Andersen Foundation and the Arvid Nilsson Foundation is gratefully acknowledged.

*Corresponding author at: Department of Micro- and Nanotechnology, Building 345Ø, room 050, DK-2800 Kongens Lyngby.

E-mail address: thomas.andresen@nanotech.dtu.dk (T.L. Andresen).

1 These Authors contributed equally to this work. tumors can potentially improve therapeutic efficacy of RT without increasing loco-regional side effects in the irradiated 43 region. ${ }^{1,2}$ Combining targeted RT and targeted drug delivery can 44 therefore increase regional tumor control. ${ }^{3}$ Moreover, liposomes 45 are flexible in regards to the selection of drugs that can be 46 encapsulated, transported and released within tumors. Lipo- 47 somes can therefore serve as optimal delivery systems for 48 targeting radiosensitizers to malignant tissue. ${ }^{1,2}$ However, 49 liposome accumulation in solid tumors has been demonstrated 50 to depend on multiple factors, including interstitial pressure, 51 tumor vasculature and perfusion. ${ }^{4-6}$ Liposome extravasation by 52 the enhanced permeability and retention (EPR) effect is primarily 53 driven by transvascular convection and their accumulation is 54 
inversely correlated to interstitial fluid pressure (IFP) and directly correlated to regional blood perfusion and leakiness. ${ }^{4,6-8}$ RT influences these parameters; however, results on the effect on tumor accumulation levels of nano-sized particles are not clear. $^{6}$

Molecular oxygen is the most important radio-sensitizer and hypoxic tumor cells are highly radio-resistant and display increased malignancy. Tumor hypoxia is generally divided in acute perfusion limited, chronic diffusion limited and anemic hypoxic. ${ }^{9}$ The nature of tumor hypoxia is closely related to vascular parameters and liposomes may therefore distribute poorly to hypoxic regions. In both experimental and clinical tumors the IFP is increased and associated with an increased malignant phenotype. ${ }^{10,11}$ RT has been associated with increased vascular leakiness, and high total radiation doses can potentially increase the extravasation of macromolecules. ${ }^{6}$ Pretreating tumors with cytotoxic agents has been identified to increase tumor blood flow and decrease IFP, potentially being the results of a reduction in tumor cell density to alleviate tumor blood vessels compressions and increase the vascular surface area which subsequently increases liposome accumulation. ${ }^{12,13}$ Following these observations the effects of RT could also mediate a beneficial effect for macromolecular extravasation by reducing cell density. ${ }^{11,14}$ Importantly, single radiation doses $>10 \mathrm{~Gy}$, are known to cause significant damage to neoangiogenic tumor vasculature and increase hypoxia and mediate significant secondary cancer cell death following vascular damage. ${ }^{15}$ On the contrary, single doses $<10$ Gy cause mild vascular damage and may potentially increase vascular perfusion and thereby decrease hypoxia after irradiation. ${ }^{15-17}$ Few studies of the effect of RT on liposome uptake have been conducted. Single-fraction irradiation had no effect on liposome uptake in human KB cancer xenografts when evaluated by gamma counting radiolabeled liposomes. ${ }^{18}$ Considering this and that important tumor dependent differences and responses may exist, we investigated the effect of single fraction radiation therapy on liposome accumulation. This was evaluated by non-invasive PET imaging in regard to i) the potential for improving liposomal drug delivery by RT $24 \mathrm{~h}$ prior to liposome administration, ii) the influence of RT on vascular tumor parameters, cellular density and necrosis and iii) locoregional liposome accumulation in hypoxic tumor regions, in a human head and neck cancer xenograft model and in a syngenic murine colon cancer model.

\section{Methods}

\section{Tumor model}

FaDu (human head and neck cancer) xenografts were established by subcutaneous injection of $\sim 5 \times 10^{6}$ cells suspended in $100 \mu \mathrm{l}$ of culture medium and Matrigel over the thigh/flank of 7 weeks old female NMRI nude mice. Tumors were allowed to grow for 12-14 days. CT26 (murine colon cancer) syngenic tumors were established by subcutaneous injection of $\sim 3 \times 10^{5}$ cells suspended in $100 \mu 1$ of culture medium over the thigh/flank of 6 weeks old female Balb/c mice.
Tumors were allowed to grow for 18 days. The National Animal 109 Experiments Inspectorate approved all study procedures. $\quad 110$

Radiolabeled liposomes

Pegylated liposomes consisting of HSPC:CHOL:DSPE- 112 PEG2k (56.5:38.2:5.3) were remote loaded with the PET isotope 113 ${ }^{64} \mathrm{Cu}^{2+}$. Briefly, $100 \mathrm{~nm} 50 \mathrm{mM}$ pegylated liposomes entrapping 114 $10 \mathrm{mM}$ DOTA were prepared as previously described. ${ }^{19} 115$ Radiolabelling was achieved by adding a volume of liposomes 116 to dried ${ }^{64} \mathrm{CuCl}_{2}$ followed by incubation at $55^{\circ} \mathrm{C}$ for $75 \mathrm{~min}$. The 117 loading efficiency was afterward evaluated by Thin Layer 118 Chromatography (Radio-TLC) and Size Exclusion Chromatog- 119 raphy (Radio-SEC), ${ }^{19}$ which showed a loading efficiency of 120 $>98 \%$ for both techniques. The liposomes were prepared at either 121 $3.3 \mathrm{mM}$ or $6.6 \mathrm{mM}$ lipid concentration and an activity 122 concentration of $62.5 \mathrm{MBq} / \mathrm{ml}$ or $125 \mathrm{MBq} / \mathrm{ml}$ (activity at the 123 time of injection) for the FaDu and CT26 tumors respectively. 124 Each animal was dosed with a volume corresponding to $22 \mu \mathrm{mol} / \mathrm{kg} 125$ and an activity of $\sim 12.5 \mathrm{MBq} /$ animal.

Radiation therapy

Mice carrying FaDu xenografts were randomized into four 128 treatment groups; non-irradiated controls $(n=11), 5$ Gy 129 $(\mathrm{n}=11), 10$ Gy $(\mathrm{n}=10)$ and 20 Gy $(\mathrm{n}=11)$. Mice carrying 130 CT26 tumors were randomized into four treatment groups; 131 non-irradiated controls $(\mathrm{n}=8), 2$ Gy $(\mathrm{n}=8), 5$ Gy $(\mathrm{n}=8)$ and 132 10 Gy $(\mathrm{n}=8)$. Radiation therapy was delivered as a single 133 fraction at a dose-rate of $1 \mathrm{~Gy} / \mathrm{min}(320 \mathrm{kV}, 12.5 \mathrm{~mA})$ using a 134 small animal irradiator (X-rad320, pXi, CT, USA). Mice were 135 irradiated in a dedicated fixation device securing that only the 136 tumor bearing leg was exposed to irradiation and the remaining 137 body shielded.

\section{MicroPET/CT imaging}

PET/CT imaging was performed on an Inveon ${ }^{\circledR}$ small animal 140 PET/CT system (Siemens Medical Systems, PA, USA) approx- 141 imately $24 \mathrm{~h}$ after completion of RT. Mice were anesthetized by 142 inhalation anesthesia ( $\sim 3 \%$ sevoflurane) and ${ }^{64} \mathrm{Cu}$-liposomes 143 injected into a tail vein. ${ }^{64} \mathrm{Cu}$-liposomes were allowed to 144 distribute for $1 \mathrm{~h}$ before commencing a 5-min PET scan (1-h 145 scan) followed by a corresponding CT scan. A similar PET/CT 146 scan (15 min acquisition) was performed after a distribution 147 period of 24 h (24-h scan). Emission data were corrected for dead 148 time and decay and attenuation correction was performed based 149 on the corresponding CT scan. PET scans were reconstructed 150 using a maximum a posteriori (MAP) reconstruction algorithm 151 $(0.815 \times 0.815 \times 0.796 \mathrm{~mm})$. Image analysis was performed 152 using Inveon ${ }^{\circledR}$ software (Siemens Medical Systems, PA, USA). 153 $3 \mathrm{D}$ regions of interest (ROIs) were manually constructed and 154 decay corrected data (\%injected dose per gram tissue $(\% \mathrm{ID} / \mathrm{g})) 155$ reported.

156

Immunohistochemistry CD31, cell density and necrosis

Immunohistochemistry (IHC) was performed on formalin- 158 fixed, paraffin-embedded $4 \mu \mathrm{m}$ tumor sections that were stained 159 with H\&E for histological evaluation and with CD31 antibodies 160 for tumor blood vessels. CD31 staining was performed by 161 
heating sections at $60{ }^{\circ} \mathrm{C}(1 \mathrm{~h})$ followed by deparaffination in xylene and rehydration. Antigen retrieval was performed by microwave-based antigen retrieval. Endogeneous peroxidase was blocked using peroxidase blocking reagent (Dako, Glostrup, Denmark) for 8 min and sections blocked in 2\% BSA for $(10$ $\mathrm{min})$. Sections were incubated with primary CD31 antibody (Abcam, diluted 1:100) in 2\% BSA (1.5 h/room temperature) followed by incubation with secondary biotinylated EnVision FLEX $^{\mathrm{TM}}$ (40 min) (Dako, Glostrup, Denmark). Tissue sections were stained with DAB $(10 \mathrm{~min})$ and counterstained with hematoxylin. Between all steps sections were rinsed in PBS.

Slides were mounted for electronic slide scanning (Axio scan, Carl Zeiss, Germany) (pixel size $0.022 \times 0.022 \mu \mathrm{m}$ ). Tumor necrosis was evaluated using the Advanced Weka segmentation plug-in for Fiji (ImageJ). The degree of necrosis in sections was determined by drawing ROIs in necrotic, background/artifacts and viable tumor region and transferring these to the trainable classifier to determine necrotic and viable areas.

Ten regions were selected on CD31 stained sections and sent for analysis of microvessel density by automated segmentation algorithm for analysis of microvessels in immunostained histological tumor sections (CAncer IMage ANalysis: http:// www.caiman.org.uk). ${ }^{20,21}$ The regions were additionally transferred to Fiji (ImageJ) for determination of nuclear density. In short, color deconvolution was performed to yield a separate hematoxylin image and the nuclei density determined by excluding fragments and artifacts by automated exclusion of structures below a cut-off size of (50 pixels $\left.^{\wedge} 2\right)$.

\section{${ }^{64} \mathrm{Cu}$-liposome autoradiography and hypoxia immunohisto- chemistry}

For analysis of intratumoral distribution of liposomes and hypoxia $\mathrm{FaDu}$ tumors (controls) were intravenously injected with the radiolabeled liposomes and these were allowed to distribute for $24 \mathrm{~h}$ before sacrificing and bleeding mice. To further study the influence of radiation therapy on intratumoral hypoxia and liposome distribution, tumors from and two CT26 tumors from each group were subjected to autoradiography and hypoxia immunohistochemistry $(26 \mathrm{~h}$ distribution period for liposomes). For hypoxia immunohistochemistry the exogenous hypoxia marker Pimonidazole (60 $\mathrm{mg}$ (kg animal $)^{-1}$ in PBS), was administered by intraperitoneal injection two hours before sacrifice. After sacrificing and bleeding animals, tumors were snap frozen and cryosectioned $(8 \mu \mathrm{m})$ in cutting media. Sections separated by at least $400 \mu \mathrm{m}$ were thaw mounted on Superfrost Plus microscopy slides. Seventeen sections from eight different FaDu tumors and five sections from included CT26 tumors were evaluated. Intratumoral distribution of ${ }^{64} \mathrm{Cu}$-liposomes was determined by exposing tumor sections to phosphor imaging screens for approximately $18 \mathrm{~h}\left(-20^{\circ} \mathrm{C}\right)$. Phosphor screen was read using a phosphor imaging system (Cyclone Plus, Perkin Elmer, MA, USA) and semi-quantitative luminescence images (pixel size $0.04 \times 0.04 \mathrm{~mm}$ ) were obtained.

Tumor sections were fixed in acetone $\left(4{ }^{\circ} \mathrm{C} / 10 \mathrm{~min}\right)$. Tissue peroxidase was quenched using peroxidase blocker (Dako, Glostrup, Denmark) and non-specific binding blocked using 2\% BSA. Pimonidazole immunohistochemistry was performed using mouse monoclonal anti-pimonidazole antibody (Hypoxyp- 218 robe, MA, USA) diluted in 2\% BSA $(1: 600)(1 \mathrm{~h})$ followed by 219 Secondary biotinylated anti-mouse antibody (40 $\mathrm{min}$ ) (Envision 220 Flex, Dako, Glostrup, Denmark). Antibody binding was 221 visualized using DAB and sections were counterstained with 222 hematoxylin $(\mathrm{H})$ and slide scanned as described above. 223

ROIs, including viable tumor regions and excluding necrotic 224 regions and artifacts were manually drawn (Fiji, ImageJ, NIH, 225 MD, USA). Automated DAB-H color deconvolution and manual 226 thresholding of pimonidazole IHC staining followed by image 227 binarization was performed in Fiji software. Autoradiography 228 images were co-registered to the corresponding pimonidazole 229 DAB-H images using a rigid co-registration algorithm 230 (MATLAB 8.4, The MathWorks, Inc., MA, United States). 231 Pimonidazole values on rescaled image (autoradiopgraphy 232 resolution) represent mean level pimonidazole positive pixels 233 on the constructed binarized image. ${ }^{64} \mathrm{Cu}$-liposome autoradiog- 234 raphy pixels were categorized into four activity levels $(0-0.25,235$ $0.26-0.50,0.51-0.75$ and $0.76-1.0)$ relative to the individual slide 236 single pixel maximum. The corresponding mean pimonidazole 237 pixel values for the four ${ }^{64} \mathrm{Cu}$-Liposome categories were 238 determined for each slide and bar-plots constructed.

Statistical analysis

Prism 7 (GraphPad Software, La Jolla, CA., USA) was used 241 for all statistical analysis. One-way or two-way ANOVA 242 analysis and Holm-Sidak multiple comparison test were applied 243 for comparisons of groups. All data are reported as mean \pm SEM 244 (standard error of mean) unless otherwise stated and a $P$-value 245 $<0.05$ considered statistically significant.

\section{Results}

${ }^{64}$ Cu-liposome PET/CT after radiation therapy

Radiation therapy was successfully delivered to all mice $24 \mathrm{~h} 249$ before administration of radiolabeled liposomes. The treatment 250 schedule was chosen to ensure that the acute effect of irradiation 251 was activity during the period of liposome distribution. To 252 evaluate the effects of the different radiation doses we extracted 253 tumor activity levels of ${ }^{64} \mathrm{Cu}$-liposome PET data from the 254 co-registered PET/CT images from the 1-h and 24-h PET/CT 255 scans. Two PET scans were performed to extract information on 256 accumulation, as intravascular liposome activity is expected to 257 dominate the 1-h PET scan and liposomes that have extravasated 258 through fenestrated tumor blood vessels the 24-h scan. PET/CT 259 images from the 24-h scans from each treatment group are 260 illustrated in Figure 1, $A-H$.

FaDu tumors displayed no significant ${ }^{64} \mathrm{Cu}$-liposome activity 262 difference between controls and treatment groups at the 1-h PET/ 263 CT (Figure 2, $A$ and $B$ ). At the 24-h PET/CT FaDu tumors 264 receiving 5 Gy and 10 Gy had significantly higher mean 265 liposome activity compared to the control group, while no 266 statistical difference was observed for the mean activity of the 20267 Gy treatment group (Figure 2, C). For the comparison of the 24-h 268 maximum activity, only the 5 Gy treatment group was 269 statistically higher than the control group (Figure 2, D). The 270 


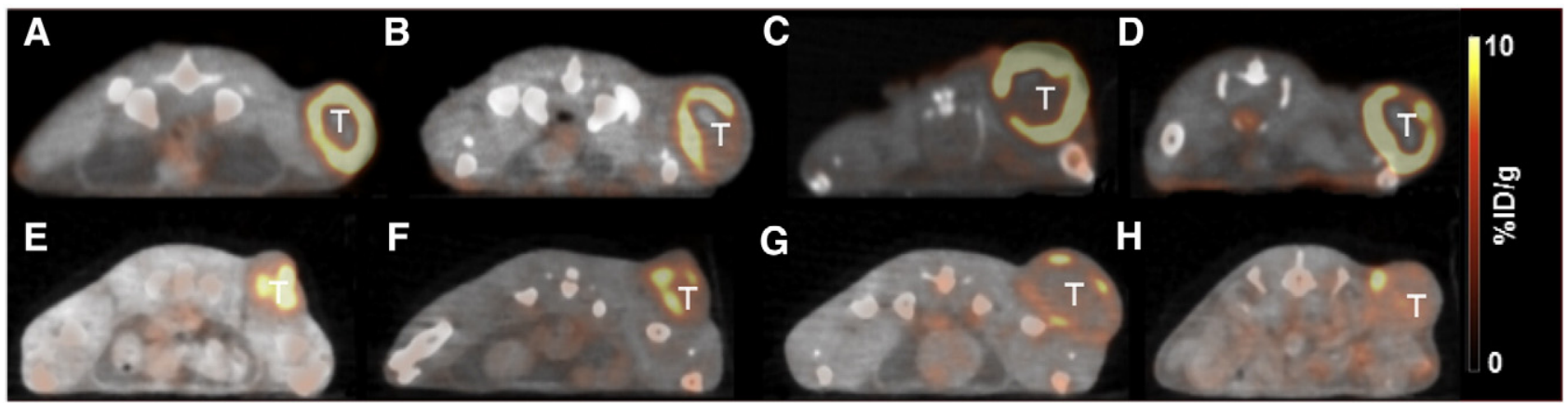

Figure 1. ${ }^{64} \mathrm{Cu}$-liposome PET/CT of FaDu xenografts (top row) (A) non-irradiated control (n = 11), (B) 5 Gy (n = 11), (C) 10 Gy (n = 10), (D) 20Gy $(\mathrm{n}=11))$ and CT26 tumors (bottom row) $\mathbf{( E )}$ non-irradiated control $(\mathrm{n}=8),(\mathbf{F}) 2 \mathrm{~Gy}(\mathrm{n}=8),(\mathbf{G}) 5$ Gy $(\mathrm{n}=8),(\mathbf{H}) 20 \mathrm{~Gy}(\mathrm{n}=8)$. (T) Tumor.

influence of radiation on liposome accumulation was further investigated in the syngenic CT26 tumors. Following the higher radiosensitivity in comparison to $\mathrm{FaDu}$ tumors, an irradiation schedule of $2 \mathrm{~Gy}, 5 \mathrm{~Gy}$ and $10 \mathrm{~Gy}$ was chosen. Interestingly, for the CT26 tumors an inverse correlation between radiation dose and liposome accumulation was observed. There was significantly higher mean activity of liposomes in the control group compared to all treatment groups at the 1-h PET scan (Figure 2, $E$ ). The control group also displayed the highest maximum activity of ${ }^{64} \mathrm{Cu}$-liposomes at the 1 -h scan although this was not significant in comparison to irradiated groups (Figure 2, F). These observations could indicate that a high level of damage was induced to intratumoral blood vessels that limit intravascular liposome blood activity. Opposite to the observations in $\mathrm{FaDu}$ tumors, the irradiated CT26 groups displayed lower activity levels in comparison to controls. This was however only statistically significant for the controls in comparison to the 5 Gy irradiated group (Figure 2, $G$ and $H$ ). Based on the conflicting results of the liposome uptake in the two included tumor models we evaluated the effect of radiation dose on tumor parameters that are expected to influence liposome accumulation.

\section{Micro vessels, nuclear density and necrosis}

The levels of intratumoral necrosis, nuclear density and micro vessels were investigated on stained tumor sections. For the $\mathrm{FaDu}$ tumor we observed a higher level of intratumoral necrosis primarily in the central parts of the tumors whereas less and more scattered distribution of necrosis was observed for the CT26 tumors. FaDu non-irradiated controls displayed a mean intratumoral necrosis level of $21.6 \%( \pm 4.5)$ while CT26 tumors only displayed $11.0 \%( \pm 1.3)$. For both tumor types the level of intratumoral necrosis increased with higher doses of radiation, except for the comparison of the $5 \mathrm{~Gy}$ FaDu group and controls. However, only the $20 \mathrm{~Gy} \mathrm{FaDu}$ group and the $5 \mathrm{~Gy}$ and $10 \mathrm{~Gy}$ CT26 groups and corresponding controls were significantly different (Figure 3, $A$ and $D$ ). As liposome accumulation is not expected to occur in devascularized non-vital necrotic regions this could explain the observed lower activity in comparison to controls for the $20 \mathrm{~Gy} \mathrm{FaDu}$ group and the irradiated groups of CT26 tumors.
Nuclear density was found to decrease with increasing 311 radiation dose. The cell density in the treatment groups all, 312 except for the $5 \mathrm{~Gy} \mathrm{FaDu}$ group, displayed significantly lower 313 cellular density compared to control groups for both tumor types 314 (Figure 3, B and E). The lower cell density is expected to 315 decrease interstitial pressure in tumors and therefore facilitate an 316 easier extravasation of liposomes. However, this was correlated 317 to an increased the overall ${ }^{64} \mathrm{Cu}$-liposome accumulation. 318 Additionally, cell density could potentially be counteracted by 319 pressure changes stimulated by radiation-induced inflammation, 320 apoptosis, necrosis and acute microvessel damage.

The micro vessel density (MVD) was investigated to identify 322 if blood vessel density could explain the observed liposome 323 activity differences. The MVD displayed no significant differ- 324 ence between FaDu groups (Figure 3, C). Interestingly, the CT26 325 control group displayed significantly higher MVD than all 326 irradiated groups (Figure 3, F). The higher mean ${ }^{64} \mathrm{Cu}$-liposome 327 activity at the 1-h PET could potentially be explained by the 328 higher microvessel density. However, for the 24-h scan this did 329 not result in significantly higher activity, whereas the $\mathrm{FaDu} 330$ tumors displayed significantly higher activity levels for the 5 Gy 331 and 10 Gy groups.

Microregional distribution of ${ }^{64} \mathrm{Cu}$-liposomes and pimonidazole 333

To investigate the potential of liposomal drug delivery system 334 to improve therapeutic control of radio-resistant hypoxic tumor 335 regions we compared the accumulation of radiolabeled lipo- 336 somes to pimonidazole hypoxia immunohistochemistry. 337 ${ }^{64} \mathrm{Cu}$-liposome autoradiographies were compared to of pimoni- 338 dazole immunohistochemistry for non-irradiated $\mathrm{FaDu}$ tumor 339 sections. The co-registration process and resizing of images 340 allowed us to include seventeen sections in the analysis. The 341 microregional pixel-to-pixel comparison of pimonidazole values 342 and corresponding categorized ${ }^{64} \mathrm{Cu}$-liposome activity level 343 identified that hypoxia decreases significantly with increasing 344 (within slide) ${ }^{64} \mathrm{Cu}$-liposome activity (Figure $4, A-D$ ). This 345 observation is important for liposome based radiosensitizer 346 therapy, as they may have limited access to important hypoxic 347 regions at least for the liposomes under investigation. Following 348 the observed influence of radiation cellular density and vascular 349 function the influence of dose on pimonidazole positive fraction 350 was investigated in CT26 tumors. We observed a significantly 351 

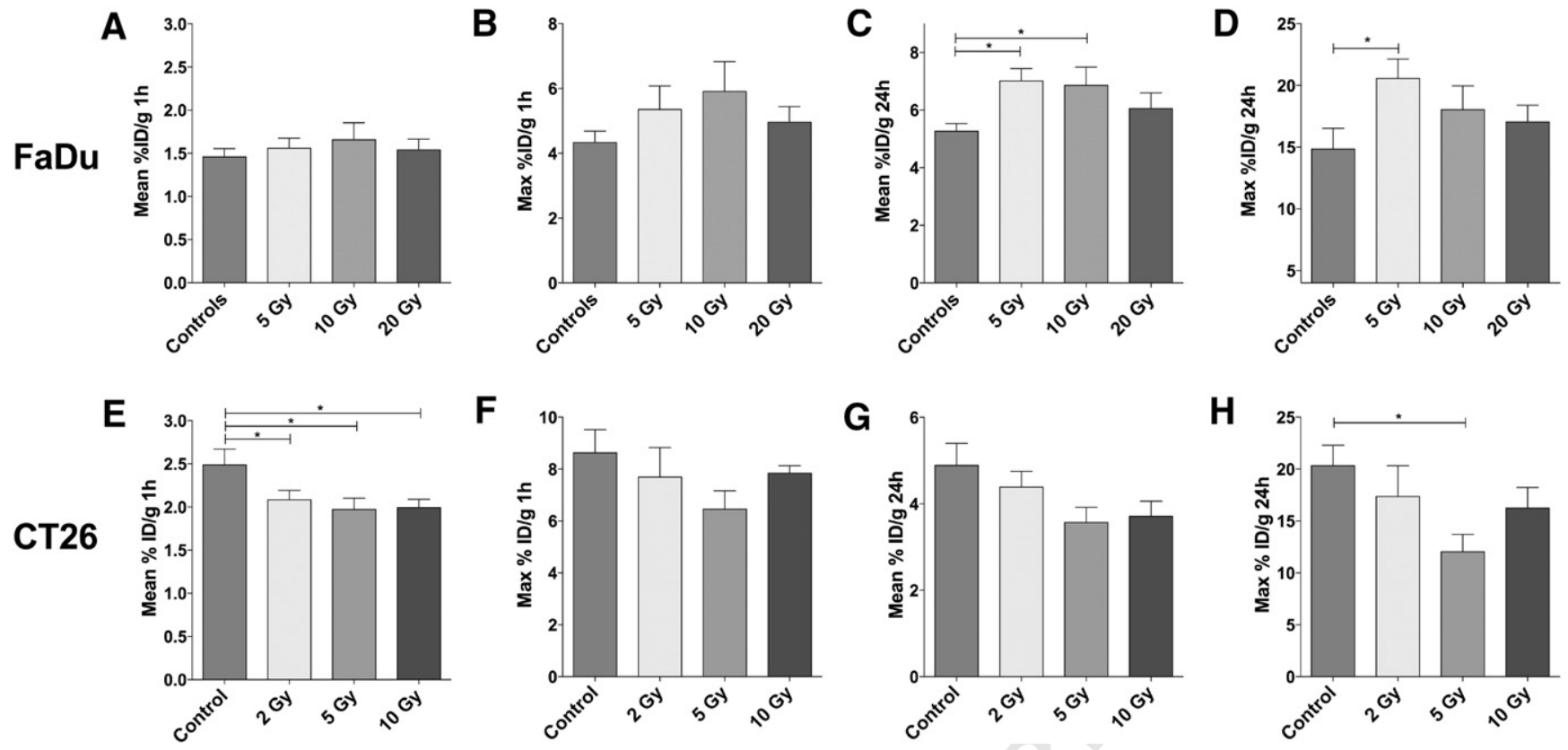

Figure 2. Tumor mean and maximum activity levels at 1-h and 24-h after injection of ${ }^{64} \mathrm{Cu}$-liposomes evaluated by PET/CT for control and treatment groups. FaDu tumors (A-D) and CT26 tumors (E-H) $(\% \mathrm{ID} / \mathrm{g} \pm \mathrm{SEM})(* P<0.05)$

lower fraction of pimonidazole positive pixel in the 2 Gy irradiated group, no difference for the 5 Gy group and a significantly increased positive fraction in the 10 Gy group relative to controls (Figure 4,E). To determine if the observed changes in tumor oxygenation could influence the distribution patterns of liposomes relative to microregional hypoxia a comparison of ${ }^{64} \mathrm{Cu}$-liposome activity level and pimonidazole was performed. The control group displayed an inverse correlation that was comparable to that of non-irradiated $\mathrm{FaDu}$ tumors (Figure 4, F). However, the 2 Gy and 5 Gy irradiated groups displayed an almost similar level of hypoxia in the different levels of ${ }^{64} \mathrm{Cu}$-liposome activity, which could indicate that these dose ranges can potentially both decrease levels of hypoxia and improve liposome accumulation in regards to hypoxic areas. This must of course be weighed against the overall accumulation of ${ }^{64} \mathrm{Cu}$-liposomes.

\section{Discussion}

The therapeutic combination of tumor targeting liposomeencapsulated radiosensitizers and radiation therapy holds great clinical potential following the dual tumor targeting properties. Notwithstanding this potential, the direct link between the parameters of central importance for liposome accumulation and the effects of radiation therapy makes the determination of optimal timing of radiation and dose and liposome administration important.

The two cancer models yielded opposite results in respect to liposomes accumulation. Whereas radiation improved accumulation in FaDu xenografts after 24-h (5 Gy and 10 Gy groups), the CT26 tumors displayed an insignificant decrease in liposome accumulation after radiation. These observations are interesting in respect to the study in human KB cancer xenografts where no 382 effect, negative or positive, on liposome uptake was observed for 383 radiation doses from 5 to 20 Gy evaluated invasively from 1 to 384 $96 \mathrm{~h}$ after irradiation. ${ }^{18}$ Both cancer models displayed an 385 increase in intratumoral necrosis and decreased cell density 386 following irradiation, both of which were most significant for the 387 CT26 tumors. Interestingly, MVD was found to respond very 388 differently to irradiation between the models. Irradiation 389 significantly decreased MVD in CT26 while FaDu tumors did 390 not display changes or patterns in relation to radiation. This was 391 also illustrated by the mean ${ }^{64} \mathrm{Cu}$-liposome activity between 392 groups after a circulation period of only 1-h. In clinical head and 393 neck squamous cell carcinomas a decrease in MVD was 394 correlated to an improved response and overall survival. ${ }^{17}$ In 395 light of this, our results indicate that the FaDu tumors represent a 396 more radio-resistant tumor and that adjuvant liposomal radio- 397 sensitizer therapy could be beneficial, at least from a dose 398 accumulation perspective for tumors maintaining a high MVD 399 during irradiation. Based on the differences in liposomes 400 accumulation and the histological analysis, accumulation 401 appeared directly dependent on a high MVD. This observation 402 is in agreement with previous publications identifying, blood 403 flow as the rate limiting step for liposome extravasation in 404 tumors with a high vascular permeability. ${ }^{25}$ However, irradiation 405 can decrease nuclear density and damage vascular structures to 406 potentially increase liposome accumulation by lowering IFP and 407 facilitating transvascular extravasation. No direct measures for 408 IFP in addition to nuclear density were performed but in a 409 previous report on irradiation of colon carcinoma xenografts 410 single fractions of 10 Gy significantly lowered IFP in tumor. ${ }^{22} 411$ From our results, the differences between MVD response and 412 comparable decrease in nuclear density between FaDu and CT26 413 tumors indicate that the MVD is the most important parameter to 414 

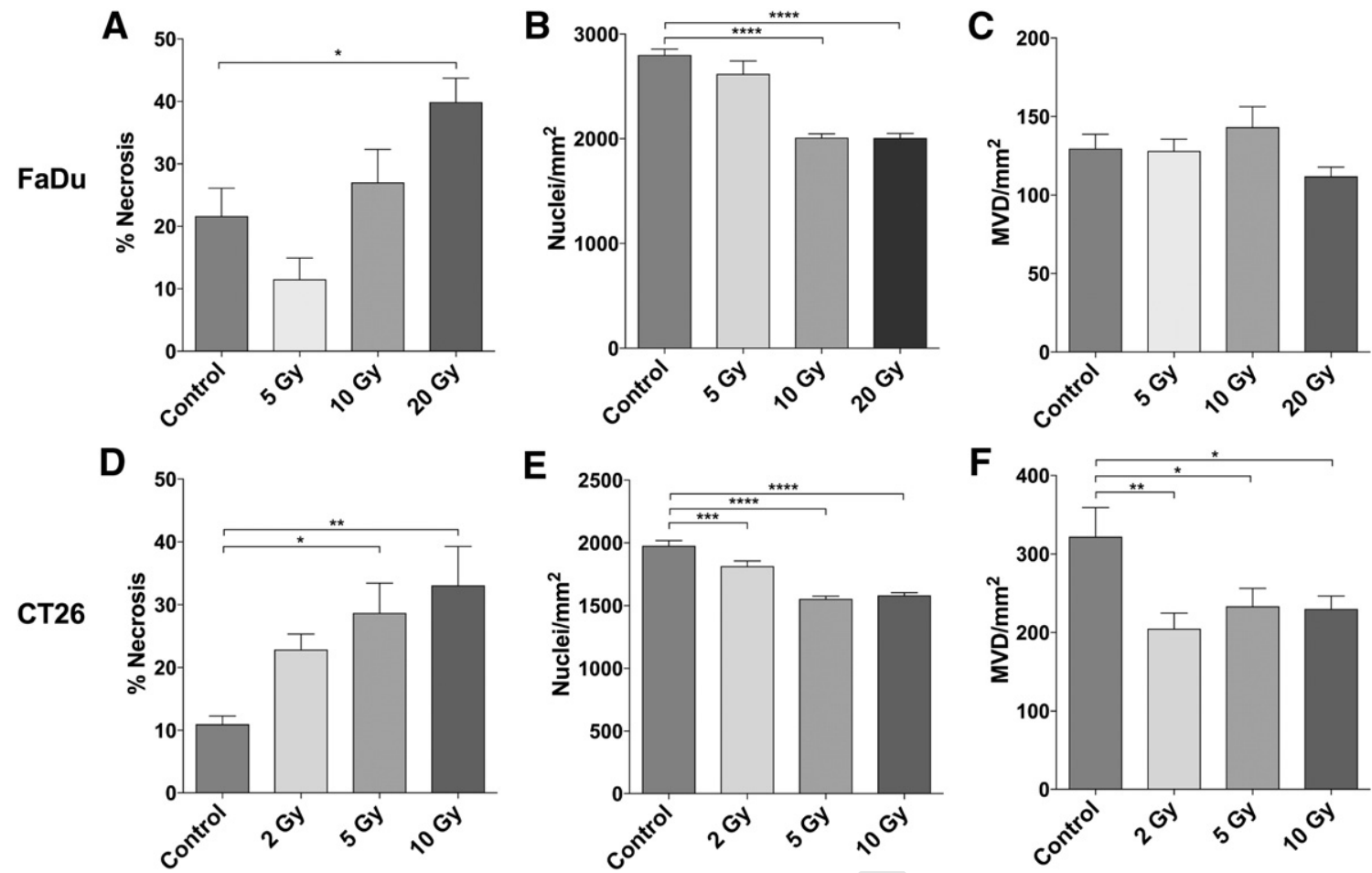

Figure 3. Immunohistochemical analysis of percentage of necrosis, nuclear counts per $\mathrm{mm}^{2}$ and microvascular density $\left(\mathrm{MVD} / \mathrm{mm}^{2}\right)$ determined by automated segmentation on tumor sections from control and treatment groups (mean $\pm \mathrm{SEM}$ ). FaDu tumors (A-C). CT26 tumors (D-F) (mean $\pm \mathrm{SEM})$. $* P \leq 0.02$, **P $\leq 0.005, * * * P<0.001, * * * * P<0.0001$.

influence liposome accumulation. Compatible results were obtained for non-small cell lung cancer patients receiving adjuvant liposomal doxorubicin to fractionated radiotherapy where MVD was associated with increased accumulation and therapeutic efficacy. ${ }^{23}$ However, the association of MVD to hypoxia could also influence this observation as discussed below. Interestingly, the increased liposome accumulation for irradiated $\mathrm{FaDu}$ tumors could also result from a decreased IFP which may improve tumor perfusion by alleviating pressure dependent collapse of intratumoral vessels. ${ }^{24,25}$ Importantly, the optimal timing of liposomal drug administration in relation to fractionated radiation remains to be determined and the reported negative impact of RT five days after irradiation indicates that timing is central for optimization of liposome accumulation. ${ }^{22}$ Based on our observations improving liposome accumulation is a balance between maintaining functional blood vessels and improving intratumoral blood flow as discussed in recent literature. ${ }^{26}$ However, the heterogeneous response of different tumor models, in regards to these parameters, highlights the value of directly quantitative PET imaging using radiolabeled liposomes.

Single doses of ( $\geq 10$ Gy) RT induce high levels of vascular damage that leads to secondary cell death when areas become deprived of oxygen and nutrients. On the other hand, fractionated low dose irradiation of tumors has been associated with improved perfusion and reoxygenation. ${ }^{15-17}$ The tumor sections evaluated from the 2 Gy and 5 Gy CT26 groups displayed less hypoxia across all levels of liposome activity, which is in line with reports on early reoxygenation after low dose irradiation. This indicates that the low dose irradiation, at least for the CT26 tumors, improves vascular perfusion and tumor oxygenation and provides the basis for a more homogeneous distribution of liposomes. The effect of 444 radiation therapy can therefore potentially also improve liposome 445 penetration and the potential of targeting liposomes that suffers 446 from inability to reach their target if trapped in the perivascular 447 regions. ${ }^{8}$ Considering the importance of hypoxia and its intricate 448 link to vascularization, optimized radiation schedules can 449 potentially improve the distribution of liposomes in radioresistant 450 hypoxic region. ${ }^{25}$ Liposomal doxorubicin has been reported to 451 increase radiosensitivity in hypoxic prostate cancer xenografts in 452 one study where clamping of the tumor-bearing leg was used to 453 induce hypoxia during RT. However, liposomes were adminis- 454 tered prior to clamping and the study therefore provides no 455 evidence that doxorubicin reaches regions of perfusion and 456 diffusion limited hypoxia, but highlights the potential of liposomal 457 chemoradiotherapy. ${ }^{27}$ Liposomal doxorubicin and cisplatin, 458 injected $16 \mathrm{~h}$ before irradiation, increased the therapeutic efficacy 459 for 4.5 Gy single dose and $9 \mathrm{~Gy} / 3$ fractions but not a single dose of 460 9 Gy radiotherapy in $\mathrm{KB}$ head and neck cancer xenografts. No 461 benefit was observed from dosing liposomes as a single compared 462 to multiple injections of the same dose and the authors were not 463 able to determine if the effects observed were truly radio- 464 sensitizing or additive, ${ }^{28}$ which highlights the importance of 465 timing to achieve a supra-additive effect chemoradiotherapy. $\quad 466$

\section{Conclusion}

The present study was conducted using a radiolabeled 468 liposome imaging system that provided quantitative data on 469 

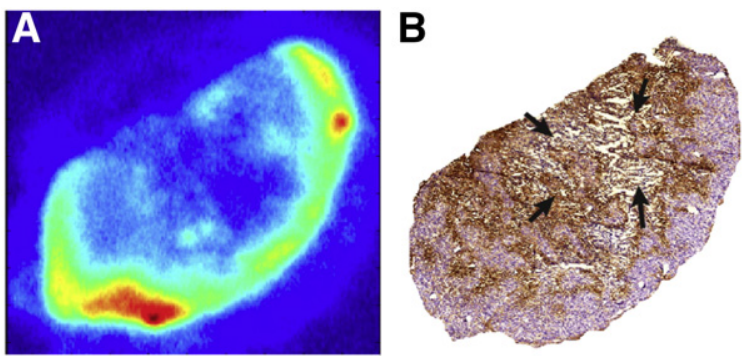

E
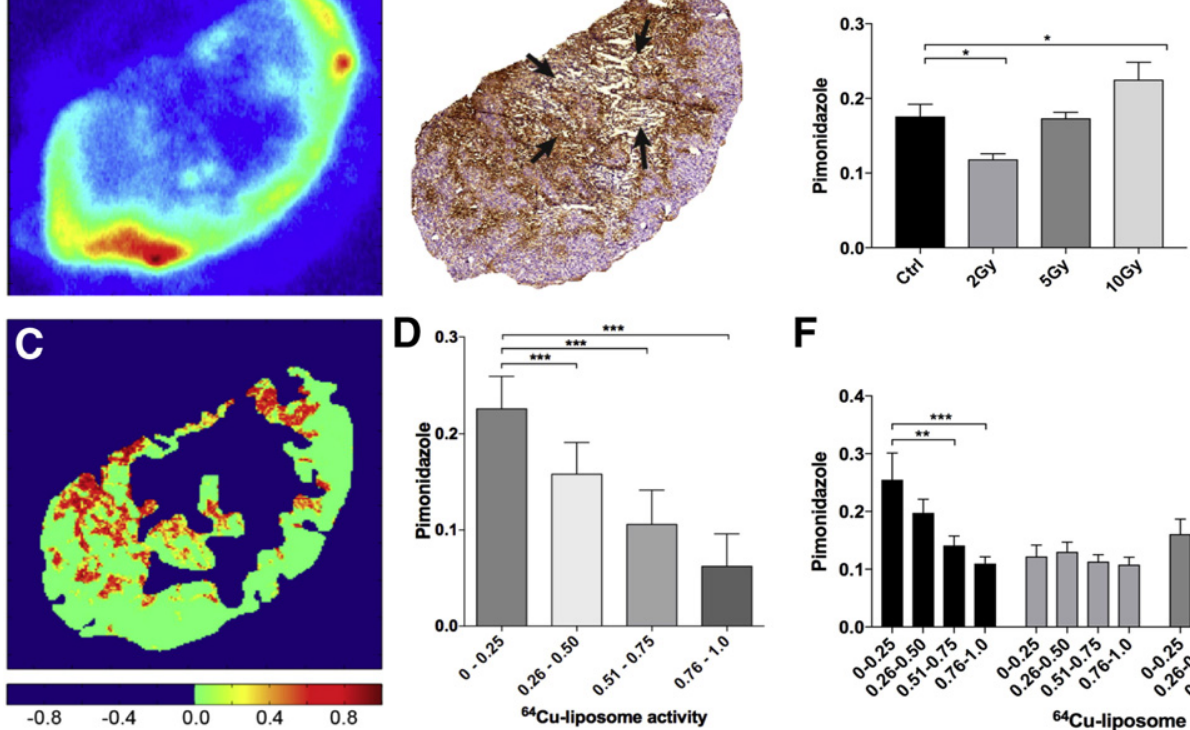

$\mathbf{F}$

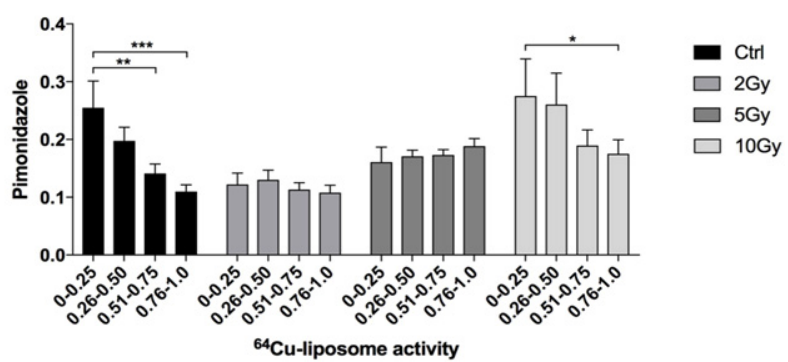

Figure 4. Microregional ${ }^{64} \mathrm{Cu}$-liposome and pimonidazole distribution evaluated on cryosectioned tumor slides. Illustrative section from a control tumor (A) ${ }^{64} \mathrm{Cu}$-liposome autoradiography, (B) pimonidazole peroxidase and hematoxylin (DAB-H) immunohistochemistry, black arrows indicate a central necrotic region, (C) interpolated pimonidazole image in false-color, color bar illustrates percent of pimonidazole positive pixels from a constructed binary pimonidazole image. (D) Bar plot illustrating the association between regional level of liposome and degree of pimonidazole hypoxia. Pimonidazole pixel values and the corresponding ${ }^{64} \mathrm{Cu}$-liposome pixel activity levels categorized according to maximum pixel activity on autoradiography (mean \pm SEM). (E) Percentage of pimonidazole positive pixels (mean \pm SEM) in tumor sections from controls and irradiated CT26 tumor sections. (F) Bar plots illustrating the association between regional ${ }^{64} \mathrm{Cu}$-liposome activity relative to section maximum and degree of pimonidazole hypoxia for the different CT26 treatment groups and controls. $(* P<0.05, * * P<0.01, * * * P<0.001)$.

liposome accumulation as a function of RT. The study identifies that RT may influence the EPR effect and liposome accumulation in a tumor and dose dependent manner. This observation emphasizes that the ${ }^{64} \mathrm{Cu}$-liposome PET imaging system may provide a theranostic tool to identify patients and treatment combinations and kinetics that may benefit from liposomal drug delivery in relation to radiation therapy. Future studies of liposomal drug delivery systems for radiosensitizers focusing on the correlation between liposome accumulation in tumor tissue as a function of RT and the therapeutic effect induced are highly warranted.

\section{References}

1. Petersen AL, Hansen AE, Gabizon A, Andresen TL. Liposome imaging agents in personalized medicine. Adv Drug Deliv Rev 2012;64:14171435.

2. R. I. Jolck, L. N. Feldborg, S. Andersen, S. M. Moghimi and T. L. Andresen, Engineering liposomes and nanoparticles for biological targeting. Adv Biochem Eng Biotechnol.125:251-80.

3. Koukourakis MI, Koukouraki S, Giatromanolaki A, Kakolyris S, Georgoulias V, Velidaki A, et al. High intratumoral accumulation of stealth liposomal doxorubicin in sarcomas - rationale for combination with radiotherapy. Acta Oncol 2000;39:207-211.

4. Li Y, Wang J, Wientjes MG, Au JL. Delivery of nanomedicines to extracellular and intracellular compartments of a solid tumor. Adv Drug Deliv Rev 2012;64:29-39.

5. Jain RK, Stylianopoulos T. Delivering nanomedicine to solid tumors. Nat Rev Clin Oncol 2010;7:653-664.
6. Multhoff G, Vaupel P. Radiation-induced changes in microcirculation Q5 and interstitial fluid pressure affecting the delivery of macromolecules 498 and nanotherapeutics to tumors. Front Oncol 2012;2:165. 499

7. Jain RK. Transport of molecules in the tumor interstitium: a review. 500 Cancer Res 1987;47:3039-3051.

8. Jain RK, Baxter LT. Mechanisms of heterogeneous distribution of 502 monoclonal antibodies and other macromolecules in tumors: signifi- 503 cance of elevated interstitial pressure. Cancer Res 1988;48:7022-7032. 504

9. Vaupel P. Hypoxia and aggressive tumor phenotype: implications for 505 therapy and prognosis. Oncologist 2008;13(Suppl 3):21-26. 506

10. Yu T, Liu K, Wu Y, Fan J, Chen J, Li C, et al. High interstitial fluid 507 pressure promotes tumor cell proliferation and invasion in oral squamous 508 cell carcinoma. Int J Mol Med 2013;32:1093-1100. 509

11. Rofstad EK, Ruud EB, Mathiesen B, Galappathi K. Associations 510 between radiocurability and interstitial fluid pressure in human tumor 511 xenografts without hypoxic tissue. Clin Cancer Res 2010;16:936-945. 512

12. Griffon-Etienne G, Boucher Y, Brekken C, Suit HD, Jain RK. Taxane- 513 induced apoptosis decompresses blood vessels and lowers interstitial 514 fluid pressure in solid tumors: clinical implications. Cancer Res 515 1999;59:3776-3782.

13. Vlahovic G, Ponce AM, Rabbani Z, Salahuddin FK, Zgonjanin L, 517 Spasojevic I, et al. Treatment with imatinib improves drug delivery and 518 efficacy in NSCLC xenografts. Br J Cancer 2007;97:735-740.

14. Tufto I, Rofstad EK. Interstitial fluid pressure, fraction of necrotic tumor 520 tissue, and tumor cell density in human melanoma xenografts. Acta 521 Oncol 1998;37:291-297.

15. Song CW, Kim MS, Cho LC, Dusenbery K, Sperduto PW. Radiobio- 523 logical basis of SBRT and SRS. Int J Clin Oncol 2014;19:570-578. 524

16. Park HJ, Griffin RJ, Hui S, Levitt SH, Song CW. Radiation-induced 525 vascular damage in tumors: implications of vascular damage in ablative 526 hypofractionated radiotherapy (SBRT and SRS). Radiat Res 527 2012; 177:311-327. 
17. Lovey J, Lukits J, Remenar E, Koronczay K, Kasler M, Nemeth G, et al. Antiangiogenic effects of radiotherapy but not initial microvessel density predict survival in inoperable oropharyngeal squamous cell carcinoma. Strahlenther Onkol 2006;182:149-156.

18. Harrington KJ, Rowlinson-Busza G, Uster PS, Vile RG, Peters AM, Stewart JS. Single-fraction irradiation has no effect on uptake of radiolabeled pegylated liposomes in a tumor xenograft model. Int $J$ Radiat Oncol Biol Phys 2001;49:1141-1148.

19. Henriksen JR, Petersen AL, Hansen AE, Frankaer CG, Harris P, Elema $\mathrm{DR}$, et al. Remote loading of (64) $\mathrm{Cu}(2+)$ into liposomes without the use of ion transport enhancers. ACS Appl Mater Interfaces 2015;7:2279622806.

20. Reyes-Aldasoro CC, Griffiths MK, Savas D, Tozer GM. CAIMAN: an online algorithm repository for cancer image analysis. Comput Methods Programs Biomed 2011;103:97-103.

21. Fliedner FP, Hansen AE, Jorgensen JT, Kjaer A. The use of Matrigel has no influence on tumor development or PET imaging in FaDu human head and neck cancer xenografts. BMC Med Imaging 2016;16:5.

22. Znati CA, Rosenstein M, McKee TD, Brown E, Turner D, Bloomer WD, et al. Irradiation reduces interstitial fluid transport and increases the collagen content in tumors. Clin Cancer Res 2003;9:5508-5513.
23. Koukourakis MI, Koukouraki S, Giatromanolaki A, Archimandritis SC, 550 Skarlatos J, Beroukas K, et al. Liposomal doxorubicin and convention- 551 ally fractionated radiotherapy in the treatment of locally advanced non- 552 small-cell lung cancer and head and neck cancer. J Clin Oncol 553 1999; 17:3512-3521.

554

24. Stapleton S, Milosevic M, Tannock IF, Allen C, Jaffray DA. The intra- 555 tumoral relationship between microcirculation, interstitial fluid pressure 556 and liposome accumulation. J Control Release 2015;211:163-170. 557

25. Stapleton S, Allen C, Pintilie M, Jaffray DA. Tumor perfusion imaging 558 predicts the intra-tumoral accumulation of liposomes. J Control Release 559 2013;172:351-357.

26. Stapleton S, Jaffray D, Milosevic M. Radiation effects on the tumor 561 microenvironment: implications for nanomedicine delivery. Adv Drug 562 Deliv Rev 2017;109:119-30.

27. Hagtvet E, Roe K, Olsen DR. Liposomal doxorubicin improves Q7 radiotherapy response in hypoxic prostate cancer xenografts. Radiat 565 Oncol 2011;6:135.

28. Harrington KJ, Rowlinson-Busza G, Syrigos KN, Vile RG, Uster PS, 567 Peters AM, et al. Pegylated liposome-encapsulated doxorubicin and 568 cisplatin enhance the effect of radiotherapy in a tumor xenograft model. 569 Clin Cancer Res 2000;6:4939-4949. 570 